\title{
Lucy - Intelligent Shopping Assistant using Python
}

Kaushal Vala, Ronak Kataria, Parth Panjabi, Amit Patel

V. T. Patel Department of Electronics and Communication CSPIT, CHARUSAT University

Changa, India

kaushalvala.ec@charusat.ac.in

katariaronak95@gmail.com

panjabipaartth@gmail.com

amitstwrt95@gmail.com

ABSTRACT: This paper focuses on a Natural Language Processing (NLP) system and Image Recognition using python and Raspberry Pi. NLP Engines are widely used in various tech domains such as surveillance, defense, advanced medical diagnosis, virtual assistant and machine learning. Open CV based image processing algorithm is applied for image recognition, facial comparison, pattern recognition, security system, object detection and deep learning. In this proposed system to implement intelligent QAS system, NLP and Image processing are integrated.

Keywords: Natural Language Processing (NLP), Image Recognition, Open CV, QAS, Text to Speech, Speech to Text, Shopping Assistant

Received: 18 November 2018, Revised 20 January 2019, Accepted 29 January 2019

DOI: $10.6025 / \mathrm{jmpt} / 2019 / 10 / 2 / 69-76$

(C) 2019 DLINE. All Rights Reserved

\section{Introduction}

With increasing varieties of products in shopping or retail domain, it becomes havoc for the customers to find their needed entity in a time. However, when dealing with a large database, searching for specific information becomes a tedious job. To overcome this difficulty, the development of an accurate Question Answering System (QAS) has been taken into the consideration.

The purpose of this paper is to create a smart QAS which can work on given commands and pre-defined database. The database trains itself whenever a new search query is fired and updates the information. User provides a search query in Natural Language to the system. The system, after processing the query, returns the most relevant answer from the dataset.

Journal of Multimedia Processing and Technologies Volume 10 Number 2 June 2019 
QAS bot works better with the structured database. This is the motivation behind the use of MySQL database. The QAS are dedicated to manipulate the predefined data, and respond to the inquired question. In this proposed system QAS chat bot will be placed at various areas as an inquiry bot for the product. The user or the customer of the Lucy will ask the question by the voice command or by typing either of the means. The user interface will reflect the response by the voice or showing it on the screen with the relevant information for the inquired product form comparing the same product. For the different customers different databases are created so that Lucy can learn the customer preferences, and can suggest the recommendations for the specific products. For e.g. If Tom ask for the watch to the bot for the specific brand and specific dial, the Lucy will ask for the type of the dial and price range, after the data is feed by the user, the Chabot will suggest the targeted product. For each customer a unique number will be created which will make the identity of the customer distinguished. The Lucy will show the filtered suggestions as per the set preferences by the customer.

The e-commerce is more preferred by the customer as they have more range in terms of designs, brand and rates, but then there comes the drawback, that customers doubts the authority of the product they are also not sure about the quality and size, whereas in Lucy the e-commerce kind of sorting in real time to the physical market i.e. shopping malls.

\section{Related Work}

As per trending automation technology such as voice engines, QAS systems are mainly based on technology such as ZigBee and other standalone devices.

Riandy Rahman Nugraha [1] implemented QAS for finding train route, apply search query and fetching departure and arrival time using hidden Markov model and speech recognizer. Udhaya Surya, Nandini K. Ishwarya M [2] have proposed a system for improving the question answering system in various open-domain and closed-domain systems used by Google, Wikipedia, AskMSR and yahoo by using web snippets. Dr. M.M Raghuvanshi [3] developed architecture of QAS for education system by detecting the question using NLP engine and query processing for implementing database search using keyword extraction. Umm-e-Laila, Muzammil Ahmad Khan, Muhmmad kashif Shaikh, Syed Annas bin Mazhar, Khalid Mehboob [4] implemented facial recognition based security system using Raspberry Pi to compare the detected face with pre- trained dataset.

\section{Proposed System}

The system consists of raspberry Pi 3 and USB webcam with USB microphone, speakers and a display. The system has been set on python, Google API library speech recognition \& Open CV.

In this paper an approach is designed of an existing system to make it more accurate by integrating NLP and image processing for QAS bot in the shopping malls for product search. The system works as a multi-vendor system, where vendor maintains the stock of the product and also let the buyers know the current trend. The vendor updates the stock in software which is synced with the system and there are multiple systems at particular area or shopping mall which are connected and synced through intranet server.

\section{Working}

When any user interacts with Lucy, it captures the face input using webcam and compare captured image with the face metadata stored in the MySQL database. If the captured input face is not recognized it asks for the detailed voice input including name, age and gender, later on it stores the metadata into database. Once the data is stored in data base Unique Identification Number is generated of each user for indexing purpose. With the use of Unique Identification Number, accessing the data of particular user from database becomes easier. Once indexing is done an optional voice input for specifying the preferences of user. If user provides preferences it upgrades the information related to that user in database.

After storing face into database, the system again takes the face input to compare with stored database. If the face is recognized than it predicts the suggestion based on previous data and collected information, for example if the age and gender of recognized user is 22 and male respectively than Lucy displays the recommended available product for youngster male. From predicted search result if user selects any of them than Lucy narrates about specifications and features of product as described y vendor as well as the shop number and product number in which the product is available. User can continue with the given recommendation or search for other product from various categories. If user sorts and filters out other product from catalog than Lucy trains

70 Journal of Multimedia Processing and Technologies Volume 10 Number 2 June 2019


database of that user. Lucy trains the database every time user searches for any product.

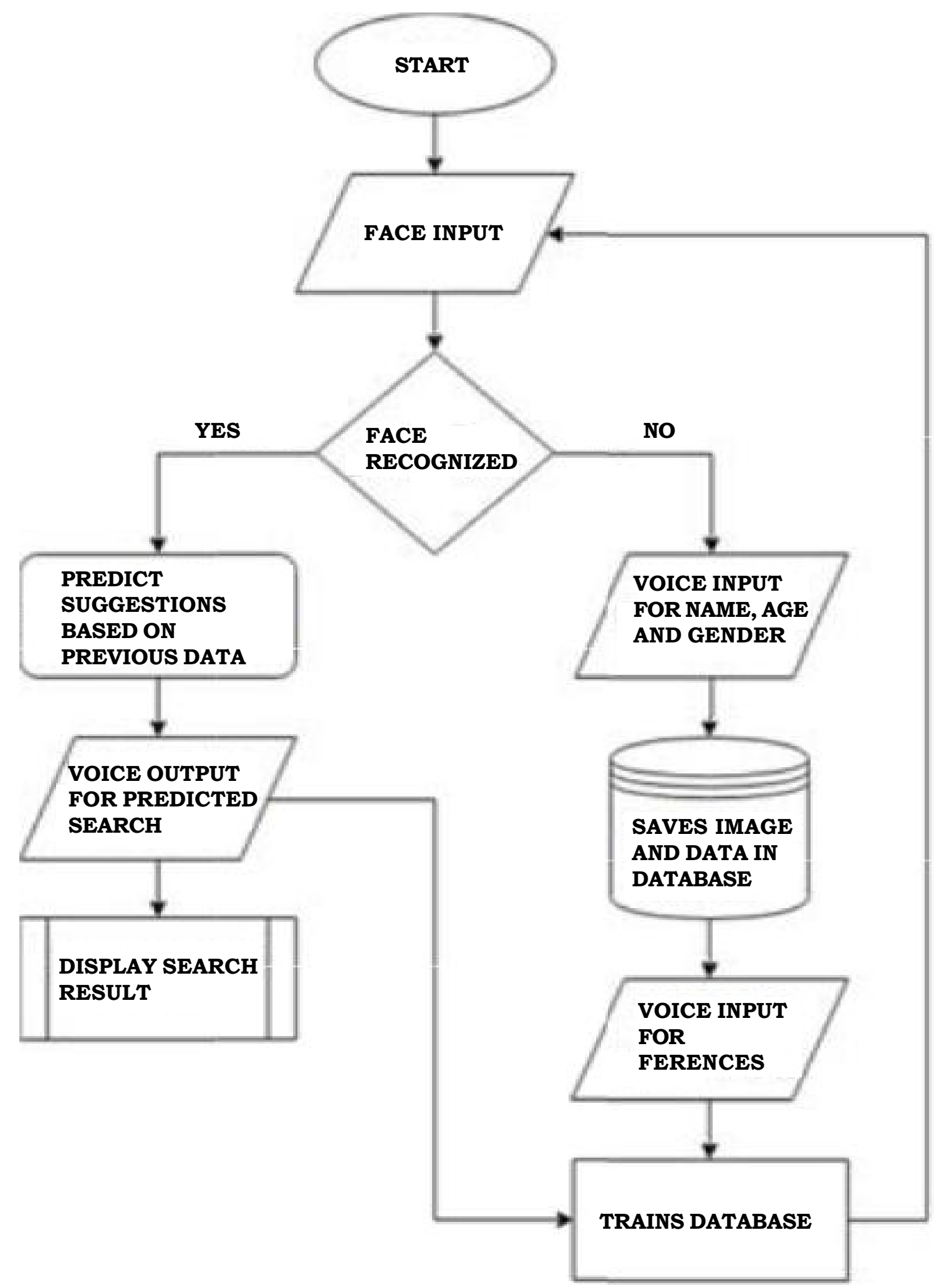

Figure 1. Flow Diagram of QAS working model 
At the end of the conversation, Lucy asks the user for reviewing the previous bought product. Vendors can read the reviews from their software for improving the user shopping experience.

\section{Experimental Results}

A face input is taken from the webcam for comparison.

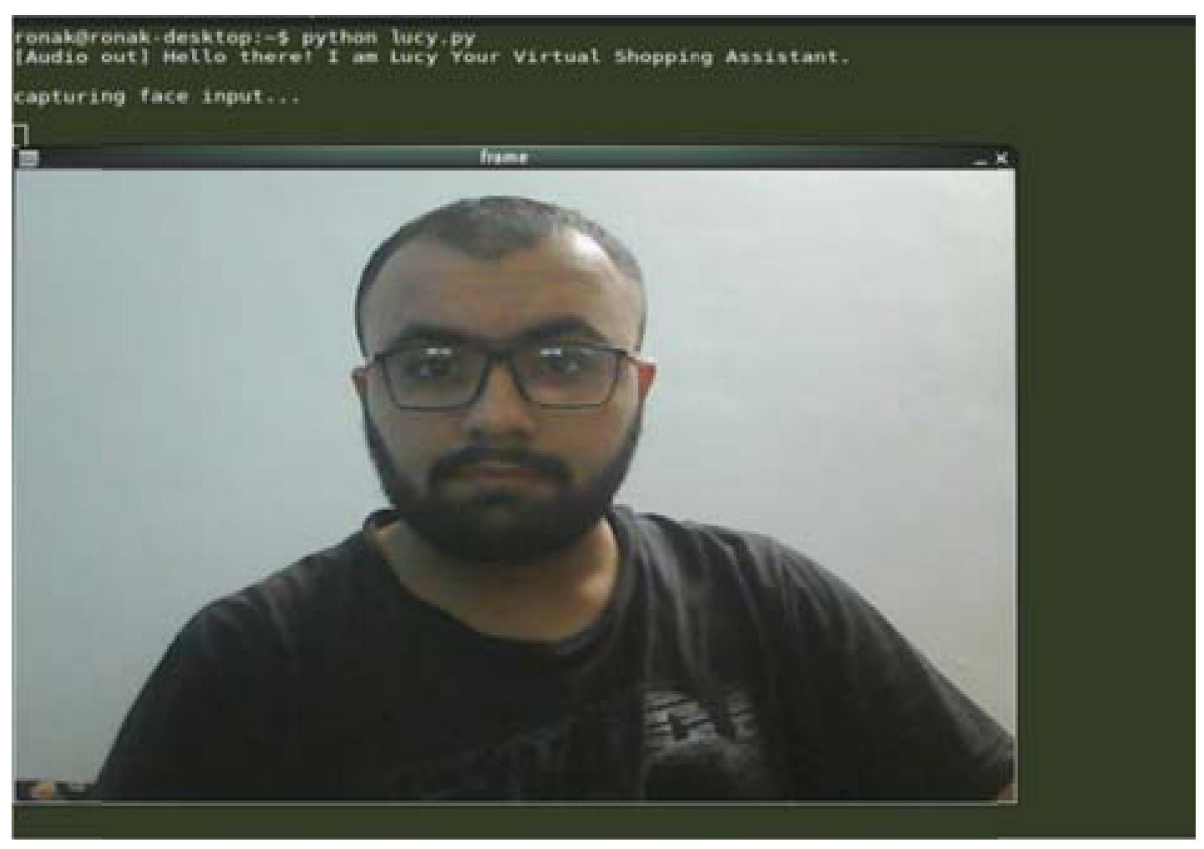

Figure 2. Capturing face input

The input face is then compared with the database for recognition. A Unique Identification Number will be generated for the user for indexing.

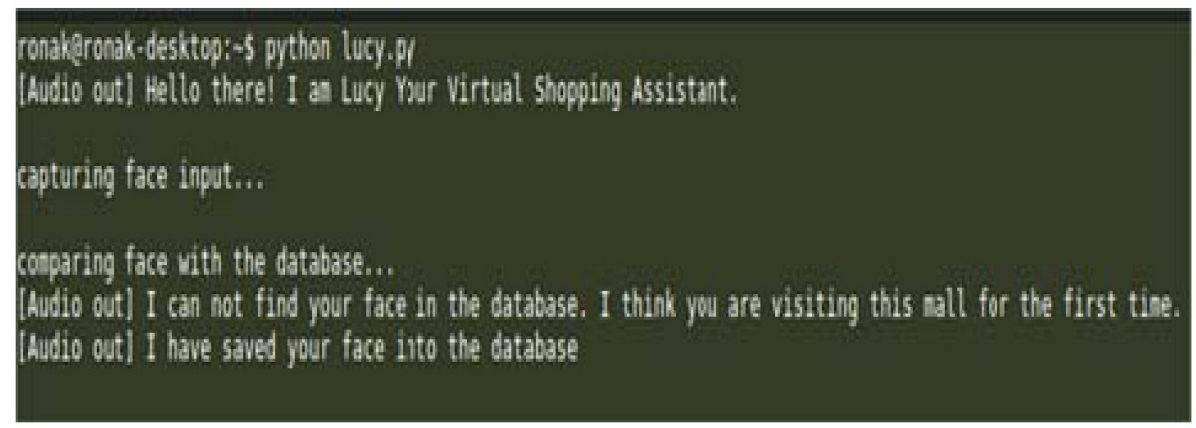

Figure 3. Comparing input with database

Name, age and gender input are taken to associate it with the stored face.

Lucy asks to input the optional preferences for creating the favorites list. If the user provides the preferences, they are saved in the database.

After the user is added in the database, Lucy can easily identify that user if he or she comes to the system again. As per the preferences given by the user, the trending products are shown if the user opts to see that.

\begin{tabular}{llllll}
\hline 72 & Journal of Multimedia Processing and Technologies & Volume & 10 & Number 2 & June \\
\hline
\end{tabular}




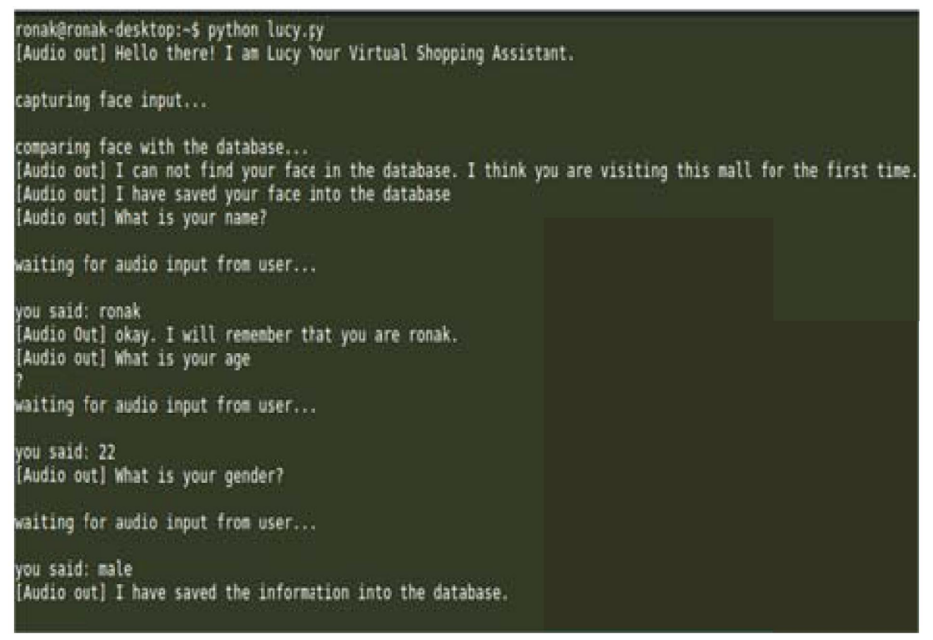

Figure 4. Saving image and related information

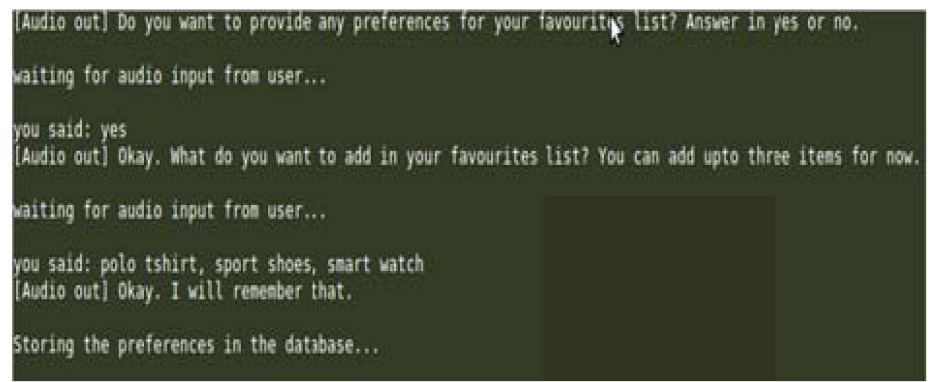

Figure 5. Storing preferences in the database

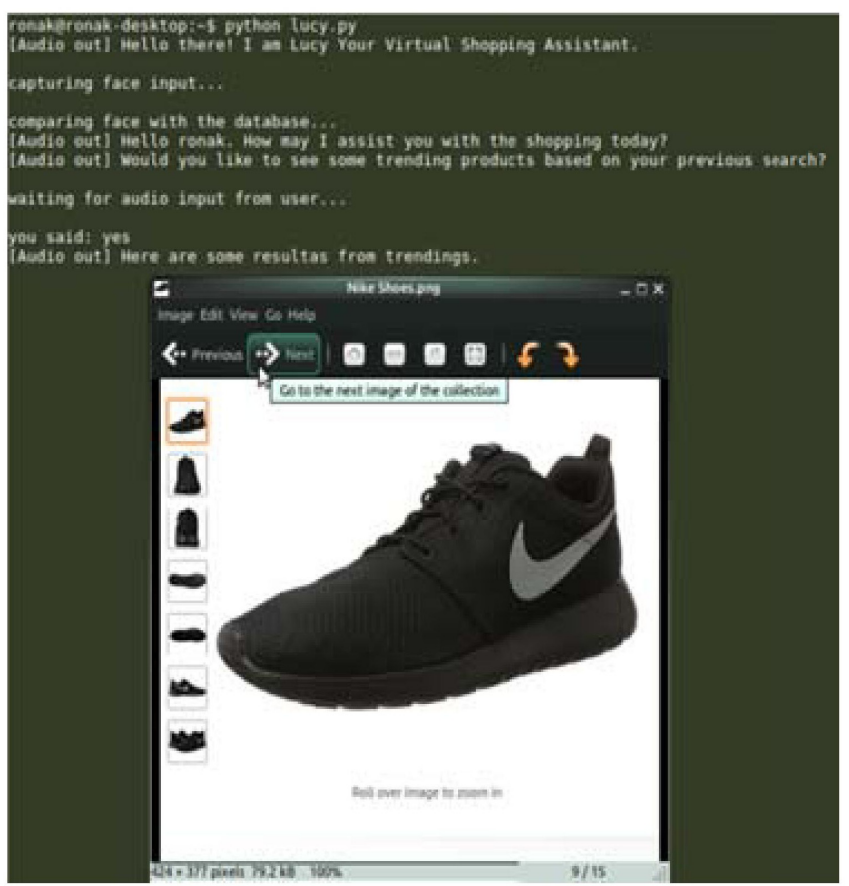

Figure 6. Recognizing and predicting 
User can scroll back and forth in the results to see more products and selects the product he or she likes.

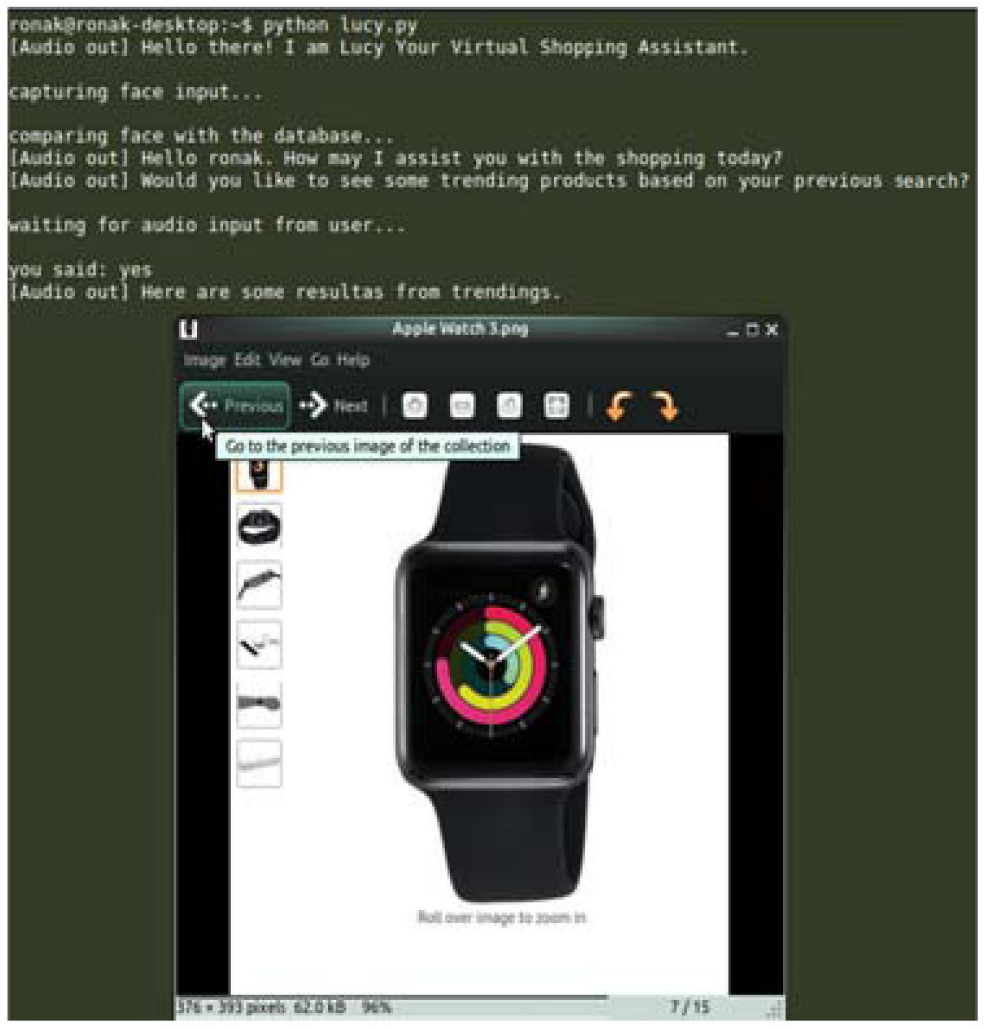

Figure 7. Recognition and predicting

Once the user selects the product from the suggestions, Lucy checks the availability and specifications of that product from the product database as per sorted by the user. Lucy also shows the shop number and product number of that product.

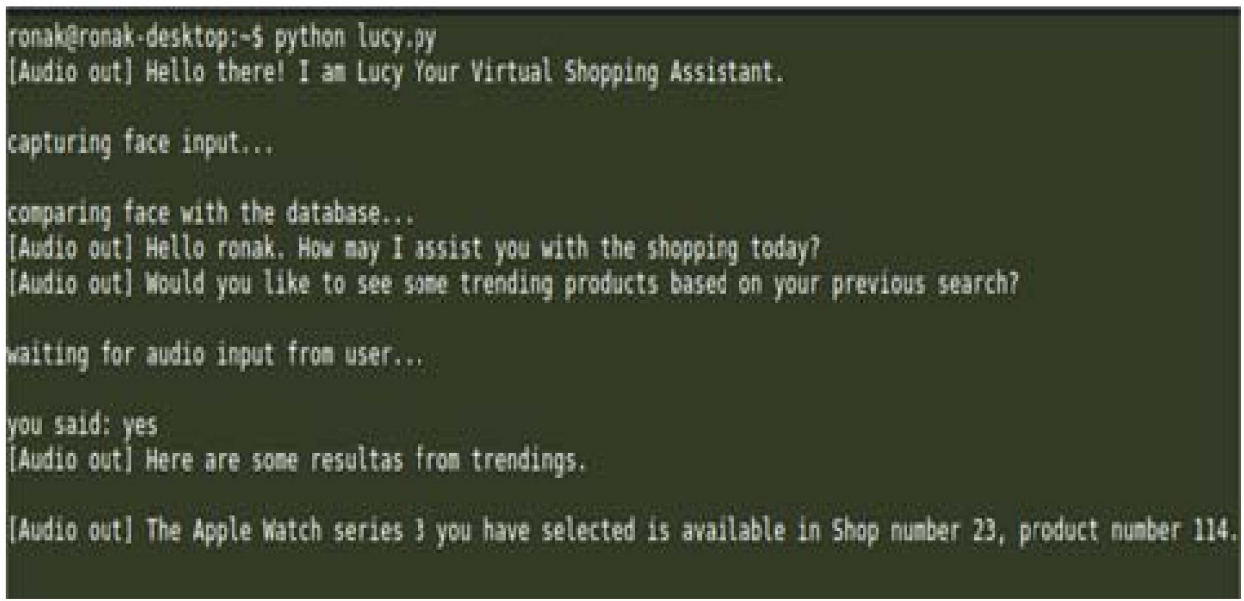

Figure 8. Information and availability of selected product

But if the user does not opt to see the trending products, Lucy will ask the user to input the search query to search the product from the catalog. Lucy compared the query with the product database and shows the availability and other details of that product.

\begin{tabular}{llllll}
\hline $74 \quad$ Journal of Multimedia Processing and Technologies & Volume & 10 & Number 2 & June & 2019 \\
\hline
\end{tabular}


Once the user completes searching of the product, Lucy asks if the user wants to review the previously bought products.

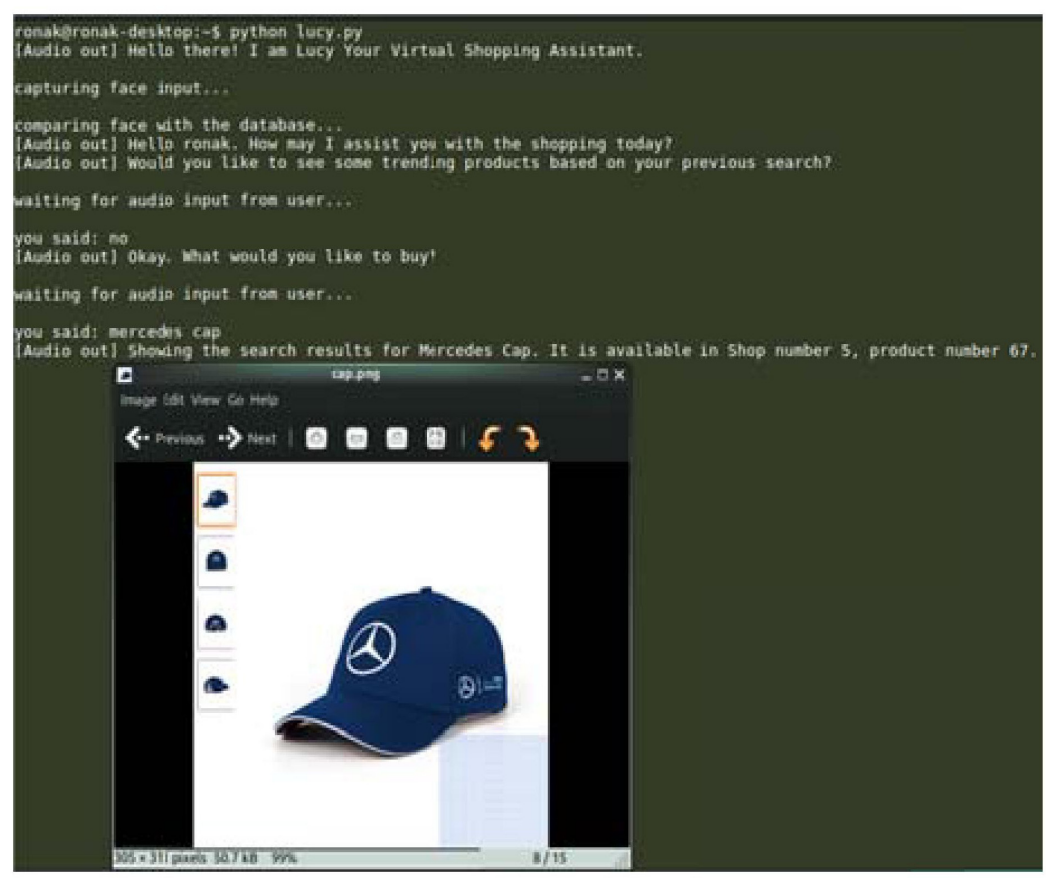

Figure 9. Upgrading database with each search

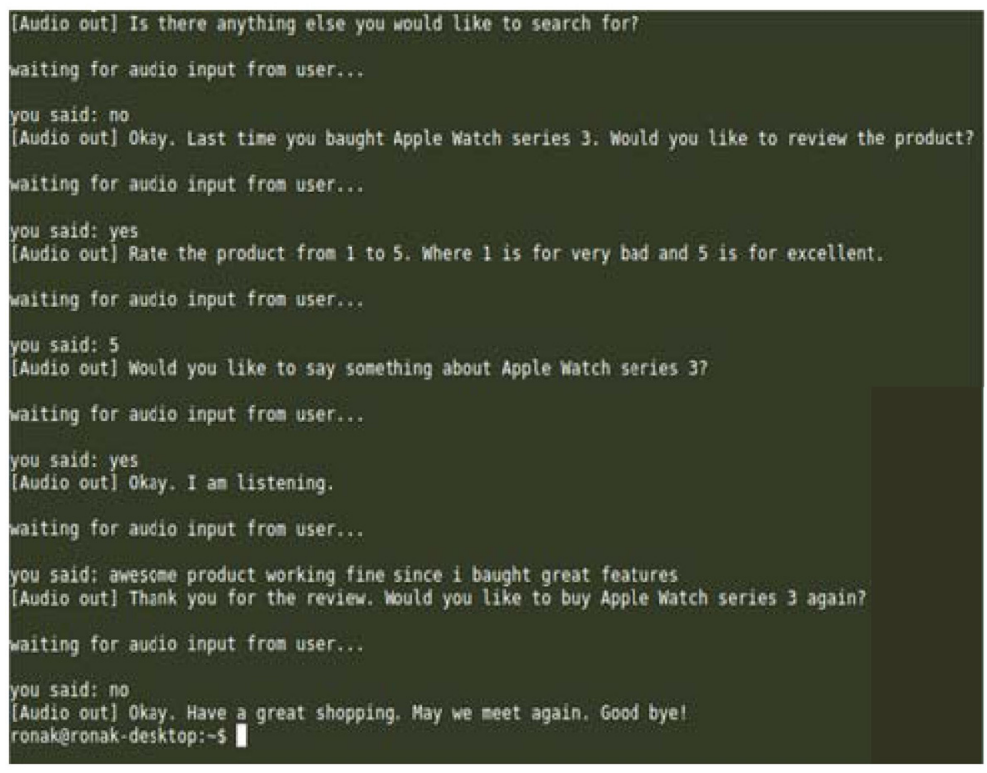

Figure 10. Storing all information

\section{References}

[1] Lestari, D., Rahman, R. (2017). A Spoken- Based Question Answering System for Train Route Service using the Frame-Based Approach in Electrical Engineering and Informatics (ICEEI), 2017 6th International Conference.

[2] Menaha, R., Udhaya Surya, A., Nandhni, K., Ishwarya, M. (2017). Question Answering System Using Web Snippets, in ISMAC (IoT in Social, Mobile, Analytics and Cloud) (I-SMAC), 2017 International Conference. 
[3] Sweta, P. (2016). Lende, Raghuwanshi, M. M. (2016). Question Answering System on Education Acts using NLP Techniques, IEEE sponsored world conference on futuristic trends in Research and Innovation for Social welfare - 2016.

[4] Umm-e-Laila, M., Ahmad Khan, Kashif Shaikh, M., Mazhar, S., Meh boob, K. (2017). Comparative analysis for a Real Time Face Recognition System Using Raspberry Pi, Smart Instrumentation, Measurement and Application (ICSIMA), 2017 IEEE 4th International Conference.

[5] Goyal, K., Agarwal, K., Kumar, R. (2017). Face Detection and Tracking Using OpenCV, Electronics, Communication and Aerospace Technology (ICECA), 2017 International Conference.

[6] Karve, S., Shende, V., Ahmed, R. (2018). A comparative analysis of feature extraction techniques for face recognition, International Conference on Communication information and Computing Technology.

[7] Mutiwokuziva, M., Chanda, M., Kadebu, P., Mukwazvure, A., Gotora, T. (2017). A Neural-network based Chat Bot, International Conference on Communication and Electronics Systems.

[8] Zhang, M., Martin, P., Powley, W., Chen, J. (2017). Workload Management in Database Management Systems: A Taxonomy, In: IEEE Transactions on Knowledge and Data Engineering. 